\title{
Processing Hyperspectral Images using Non-Linear Least Square Algorithm as an Optimization Method for Tensor Decomposition Model
}

\author{
Ankit Gupta \\ M.Tech Student \\ Maharishi Markandeshwar \\ University Mullana, India
}

\author{
Nishi Goel \\ M.Tech Student \\ Maharishi Markandeshwar \\ University Mullana, India
}

\author{
Ashish Oberoi \\ Associate Professor \\ Maharishi Markandeshwar \\ University Mullana, India
}

\begin{abstract}
Due to large size and huge availability of unwanted or missing information in hyperspectral image, development of data effective compression and denoising methods is of prior importance. Compression removes unmeaningful information and thereby reducing data which ultimately leads to noise free image. This study deals with execution of two lossless decomposition methods Low Multi-linear Rank Approximation, four types of Block Term Decomposition to the input image cube to make it noise free using non-linear least square method as an optimization method and their performance were assessed. BTD $\left(\mathrm{L}_{\mathrm{r}}, \mathrm{L}_{\mathrm{r}}, 1\right)$ was selected as the best tensor algorithm based on residual error and frobenius norm value with a limitation that the image cube to be processed by the method should have good spatial resolution.
\end{abstract}

\section{General Terms}

Remote Sensing

\section{Keywords}

Hyperspectral imaging, Data Compression, Tensor decomposition models, Low Multi-linear Rank Approximation, Block Term Decomposition, Frobenius Norm.

\section{INTRODUCTION}

Hyperspectral imaging being a remote sensing technology provides rich source of information for detailed analysis. Hyperspectral data suffers from spatial and spectral blurriness due to gaseous disturbances present between sensors and earth surface [7].Hyperspectral image processing allows removal of un-meaningful by preserving useful information and pure spectra identification [9].Useful information in the image may be distorted due the information resulting from unknown sources. Algorithms designed and developed forprocessing of the image cube share the common ground but have different forms due to different degrees of target information [19].Data effective compression methods arean active area of research for the enhancement of tools and techniques in remote sensing technology. Compression methods which are lossy allow noisy data to be removed from the image whereas lossless data compression preserves all data [1].Researchers are more focusing on lossless compression methods as far as tensor decomposition is concerned. Since hyperspectral image is multivariate, multi-way arrays can also be used for data compression. Multi-way arrays having three or more dimensions or mode are called high order tensor. It can be used for deducing noise free image cube from the noised one. Mathematics used for tensor tools and analytics is multi-linear algebra and is a high order generalization of linear algebra.
Tensor models such as Canonical Polyadic Decomposition [2], Low Multi-linear Rank Approximation [12], and Block Term Decomposition [8]decompose hyperspectral image in to low rank cubes in order to reduce it size by removing unmeaning information and missing entries with the advantage of integrating spatial-spectral domain.CPD ,LMLRA reduces the original tensor into sum of rank-1 tensors, tensor of lower dimension multiplied with factor matrices respectively.BTD is the combination of both, it decomposes the tensor into series of core tensors along with corresponding factor matrices i.e. sum of low multi-linear rank terms. Choosing the size of low rank tensor is a tedious task because there is no such a criterion or algorithm to choose the size of low rank tensors. These tensor models use non-linear least square as an optimization method for tensor decomposition. This deals with application of low multi-linear rank approximation (LMLRA), Block Term Decomposition (BTD) models.BTD is further sub divided in to three types based on type of dimensions of resultant tensor BTD $(\mathrm{L}, \mathrm{M}, \mathrm{N}), \mathrm{BTD}(\mathrm{Lr}, \mathrm{Lr}, 1), \mathrm{BTD}(\mathrm{L}, \mathrm{M},-)$ which will be discussed in further sections.

Rest of the paper is organized as follows: Section2, 3, 4 and 5 discusses mathematical formulation of Tensor decompositions, related work, evaluation results and conclusion respectively.

\section{TENSOR DECOMPOSITON MODELS}

Hitchcock discovered tensor decompositions in 1927[2].Tensor decomposition is considered to be the high order generalization of singular value decomposition [12]. It deals with factorization of tensor in to core tensor and factor matrices. Main focus while designing the decomposition algorithm is there should not be any loss of information during decomposition [6].

\subsection{Notations}

Hyperspectral image can be represented by third order tensor denoted by $\mathcal{H} \in A^{I_{1} X I_{2} X I_{3}}$ having three modes $\mathrm{I}_{1}, \mathrm{I}_{2}, \mathrm{I}_{3}$ whose elements are represented as $e_{i_{1} i_{2} i_{3}}$ with $\mathrm{i}_{\mathrm{j}=1,2,3}$. p denotes scalars and vector is denoted by $\mathbf{p}$. Matrices are represented by capital boldface letters i.e. $\mathbf{P}$ with $i$ as the row index and $\mathbf{j}$ as the column index. Element of matrix $\mathbf{P}$ is denoted by $a_{\mathrm{ij}}$. Kronecker product and Khatri-Rao product is denoted by $\odot$ , $\otimes$ and $^{T},{ }^{\dagger}$ denotes transpose and conjugate inverse respectively. 
Kronecker product for two matrices A and B denoted by

$$
\mathbf{P} \otimes \mathbf{Q}=\left(\begin{array}{ccc}
p_{11} \boldsymbol{Q} & \cdots & p_{1 n} \boldsymbol{Q} \\
\vdots & \ddots & \vdots \\
a_{m 1} \boldsymbol{Q} & \cdots & a_{m n} \boldsymbol{Q}
\end{array}\right)
$$

Suppose $\mathrm{P}=\left[\mathrm{P}_{1} \ldots \mathrm{P}_{\mathrm{R}}\right]$ and $\mathrm{Q}=\left[\mathrm{Q}_{1} \ldots \mathrm{Q}_{\mathrm{R}}\right]$ are two apportioned matrices. ThenKhatri-Rao product for matrices $\mathrm{P}$ and $\mathrm{Q}$ is defined by:

$$
P \odot Q=\left(P_{1} \otimes Q_{1} \ldots P_{R} \otimes Q_{R}\right)
$$

Frobenius norm for tensor $\mathcal{H} \in A^{I_{1} X I_{2} X I_{3}}$ is given by:

$$
\|\mathcal{H}\|=\operatorname{sqrt}\left(\sum_{i=1}^{I} \sum_{j=1}^{J} \sum_{k=1}^{K}\left|\hbar_{i j k}\right|^{2}\right)
$$

\subsection{Low Multi-linear Rank Approximation}

Tucker introduced LMLRA in 1963 [4] which was further modified by Tucker and Levin.It is a kind of high-order PCA which decomposes a tensor into core tensor multiplied by factor matrices

The LMLRA decomposition for a tensor $\mathcal{H} \in A^{I_{1} X I_{2} X I_{3}}$ is mathematically defined by:

$$
\mathcal{H}=\mathcal{X} \cdot{ }_{1} P \cdot{ }_{2} Q \cdot{ }_{3} S
$$

where is a core tensor of size $(\mathrm{L}, \mathrm{M}, \mathrm{N})$ and $\mathbf{P}, \mathbf{Q}, \mathbf{S}$ are factor matrices.

Core tensor and factor matrices are mathematically represented as:

$$
\begin{gathered}
X \in \mathcal{H}^{L \times M \times N} \\
P \in \mathcal{H}^{I \times L} \\
Q \in \mathcal{H}^{J \times M}
\end{gathered}
$$

$$
S \in \mathcal{H}^{K \times N}
$$

Matrix representation of eq. 3 can be written as:

$$
\begin{aligned}
& \mathcal{H}_{J K \times I}=\mathrm{Q} \otimes \mathrm{s} \cdot \mathcal{D}_{M N \times L} \cdot P^{T} \\
& \mathcal{H}_{K I \times J}=\mathrm{s} \otimes \mathrm{P} \cdot \mathcal{D}_{N L \times M} \cdot Q^{T} \\
& \mathcal{H}_{I J \times K}=\mathrm{P} \otimes \mathrm{Q} \cdot \mathcal{D}_{L M \times N} \cdot S^{T}
\end{aligned}
$$

Principal components among each mode can be factor matrices $\mathbf{P}, \mathbf{Q}$, Sand core tensor indicates the interaction level among different components.Factor matrices are computed as right singular vectors associated with non-zero singular values of matrices $\mathcal{H}_{I K \times I} \mathcal{H}_{K I \times J} \mathcal{H}_{I J \times K}$. Rank of these matrices relates to the value of $\mathrm{L}, \mathrm{M}$, and $\mathrm{N}$ for core tensor.

\subsection{Block Term Decomposition}

In BTD tensor is represented as sum of low rank tensors [5]. It is a hybrid variety of tensor models combining two basic tensor models. BTD combines CPD and tucker decomposition models.BTD is further divided into three parts which will be discussed in further sub sections.

2.3.1 BTD $\left(L_{r}, L_{r}, 1\right)$

It decomposes a tensor $\mathcal{H} \in A^{I_{1} X I_{2} X I_{3}}$ into sum of $(\mathrm{Lr}, \mathrm{Lr}, 1)$ rank terms where $r \leq R[22]$.Mathematically it can be shown as:

$$
\mathcal{H}=\sum_{r=1}^{R}\left(\boldsymbol{P}_{r} \cdot \boldsymbol{Q}_{r}^{T}\right) \circ S_{r}
$$

$\boldsymbol{P}_{r} \in \boldsymbol{A}^{\mathrm{I} \times L_{r}}, \boldsymbol{Q}_{r} \in \boldsymbol{A}^{\mathrm{J} \times L_{r}}$ areL $_{\mathrm{r}}$ rank matrices where $1 \leq r \leq R$.

Let $\mathbf{P}=\left[\mathbf{P}_{1} \ldots \mathbf{P}_{\mathrm{r}}\right], \mathbf{Q}=\left[\mathbf{Q}_{1} \ldots \mathbf{Q}_{\mathrm{r}}\right]$ and $\mathbf{S}=\left[\mathbf{S}_{1} \ldots \mathbf{S}_{\mathrm{r}}\right]$, then decomposition in matrix form can be written as

$$
\begin{aligned}
& \mathcal{H}_{I J \times K}=\left[\left(P_{1} \odot_{s} Q_{1}\right) 1_{L_{1}} \ldots\left(P_{R} \odot_{s} Q_{R}\right) 1_{L_{R}}\right] \cdot S^{T} \\
& \mathcal{H}_{J K \times I}=(\boldsymbol{Q} \odot \boldsymbol{S}) \cdot \boldsymbol{P}^{T} \\
& \mathcal{H}_{K I \times J}=(\boldsymbol{S} \odot \boldsymbol{P}) \cdot \boldsymbol{Q}^{T}
\end{aligned}
$$

(Lr, Lr, 1) rank terms are tri-linear in factor matrices $\mathbf{P}, \mathbf{Q}$, Swhich means updating one matrix given two matrices is a linear least square problem.

\subsection{2 $\operatorname{BTD}(L, M, N)$}

This type of BTD decomposes a tensor $\mathcal{H} \in A^{I_{1} X I_{2} X I_{3}}$ into sum of rank (L, M, N) terms[22] as follows:

$\mathcal{H}=\sum_{r=1}^{R} X_{r} \cdot{ }_{1} \boldsymbol{P}_{r} \cdot{ }_{2} \boldsymbol{Q}_{r} \cdot{ }_{3} \boldsymbol{S}_{r}$

$\chi_{r} \in \mathcal{H}^{L \times M \times N}$ is full rank and

$\boldsymbol{P}_{\boldsymbol{r}} \in \mathcal{H}^{I \times L}, \boldsymbol{Q}_{\boldsymbol{r}} \in \mathcal{H}^{J \times M}$ and $\boldsymbol{S}_{\boldsymbol{r}} \in \mathcal{H}^{K \times N}$ are full column rank.IfP $=\left[\mathbf{P}_{1} \ldots \mathbf{P}_{\mathrm{r}}\right], \mathbf{Q}=\left[\mathbf{Q}_{1} \ldots \mathbf{Q}_{\mathrm{r}}\right]$ and $\mathbf{S}=\left[\mathbf{S}_{1} \ldots \mathbf{S}_{\mathrm{r}}\right]$ Then decomposition in matrix form can be written as:

$\mathcal{H}_{J R \times l}=(Q \odot S)$.blockdiag $\left(\left(X_{1}\right)_{M N \times L, \cdots,}\left(X_{R}\right)_{M N \times L}\right) \cdot P^{T}$

$\mathcal{H}_{R[\times]}=(S \odot P) \cdot \operatorname{blockdiag}\left(\left(X_{1}\right)_{M N \chi L}, \ldots,\left(X_{R}\right)_{M N \times L}\right) \cdot Q^{T}$

$\mathcal{H}_{I J \times R}=(P \odot Q)$, blockdiag $\left(\left(X_{1}\right)_{M N \times L}, \cdots,\left(X_{R}\right)_{M N X L}\right) S^{T}$ 
Vector representation of $\mathcal{H}$ is given as:

$$
\mathcal{H}_{I J K}=(\boldsymbol{P} \odot Q \odot \boldsymbol{S}) \cdot\left(\begin{array}{c}
\left(X_{1}\right)_{M N \times L} \\
\vdots \\
\left(X_{R}\right)_{M N \times L}
\end{array}\right)
$$

One matrix is conditionally updated by keeping two matrices and core tensor fixed. Same is the case for updating core tensor i.e. core tensor is updated by keeping all the three factor matrices fixed.

\subsection{3 $B T D(L, M, \cdot)$}

$\mathrm{BTD}(\mathrm{L}, \mathrm{M}, \cdot)$ decomposes the tensor $\mathcal{H} \in A^{I_{1} X I_{2} X I_{3}}$ into sum of (L, M,-) terms[22]. Mathematically it can be written as:

$$
\mathcal{H}=\sum_{r=1}^{R} \boldsymbol{S}_{r} \cdot{ }_{1} \boldsymbol{P}_{r} \cdot{ }_{2} \boldsymbol{Q}_{r}
$$

$$
\boldsymbol{S}_{r} \in \mathcal{H}^{L \times M \times K}
$$

$\boldsymbol{P}_{\boldsymbol{r}} \in \mathcal{H}^{I \times L}(I \geq L)$

$\boldsymbol{Q}_{\boldsymbol{r}} \in \mathcal{H}^{J \times M} \quad(J \geq M)$ are full column rank, $r \leq R$. In terms of matrix representation with $\mathbf{P}=\left[\mathbf{P}_{1} \ldots \mathbf{P}_{\mathrm{r}}\right], \mathbf{Q}=\left[\mathbf{Q}_{1} \ldots \mathbf{Q}_{\mathrm{r}}\right]$ and $\mathbf{S}=$ $\left[\mathbf{S}_{1} \ldots \mathbf{S}_{\mathrm{r}}\right]$ above equation can be written as:

$$
\mathcal{H}_{I J K}=(\boldsymbol{P} \odot \boldsymbol{Q}) \cdot\left(\begin{array}{c}
\left(S_{1}\right)_{L M \times K} \\
\vdots \\
\left(S_{R}\right)_{L M \times K}
\end{array}\right)
$$

$$
\mathcal{H}_{K I \times J}=\left[\left(S_{1} \cdot{ }_{1} P_{1}\right)_{K I \times M} \ldots\left(S_{R} \cdot{ }_{1} P_{R}\right)_{K I \times M}\right] \cdot Q^{T}
$$

$$
\mathcal{H}_{J K \times I}=\left[\left(S_{1} \cdot{ }_{2} Q_{1}\right)_{K I \times M} \ldots\left(S_{R} \cdot{ }_{2} Q_{R}\right)_{K I \times M}\right] . P^{T}
$$

\subsubsection{Non-Linear Least Square}

Besides alternating least square methods non-linear least square methods can also be used for optimization during tensor decompositions. In this study we have used two nonlinear least square methodsGauss-Newton, LevenbergMarquardt [20]. These methods deals with approximation of residual tensor using linear model

$$
m_{k}^{\mathcal{H}}\left(\begin{array}{l}
c \\
p
\end{array}\right) \triangleq \operatorname{vec}\left(\mathcal{H}\left(\begin{array}{c}
c \\
z_{k}
\end{array}\right)\right)+\frac{\partial \operatorname{vec}\left(\mathcal{H}\left(\begin{array}{c}
c \\
z_{k}
\end{array}\right)\right)}{\partial_{Z^{T}}^{C}} p
$$

Where complex jacobian at ${ }_{z_{k}}^{c}$ is the partial derivative term in equation 20 which is a generalization of complex gradient [21].Now for the modified quadratic model of the objective function, $m_{k}^{\mathcal{H}}$ is used as follows:

$$
m_{k}^{h}\left(\begin{array}{l}
C \\
p
\end{array}\right) \triangleq \frac{1}{2}\left\|m_{k}^{\mathcal{H}}\left(\begin{array}{l}
C \\
p
\end{array}\right)\right\|^{2}+\frac{\lambda_{k}}{2}\|p\|^{2} \mid
$$

Where Levenberg-Marquardt regularization parameter is $\lambda_{k}$ which impacts the $\mathrm{p}$ parameter length and direction for minimization of $m_{k}^{h} \cdot \lambda_{k}$ is zero for Gauss Newton method for all $\mathrm{k}$ and for controlling length and direction of the step trust region framework can be used. If $\mathcal{H}_{B T D}$ is analytic in its argument then working on the half complex jacobian is sufficient since identically $\frac{\partial \operatorname{vec}\left(\mathcal{H}_{B T D}\right)}{\partial \bar{z}^{T}}=0$.Hence minimization of quadratic model byLevenberg-Marquardt step is given as:

$p_{k}^{*}=-\left[\begin{array}{c}J_{k} \\ \sqrt{\lambda_{k}}\end{array}\right]^{\dagger}\left[\begin{array}{c}\operatorname{vec}\left(\mathcal{H}_{B T D}\left(z_{k}\right)\right) \\ 0\end{array}\right]$

Where at $\mathrm{z}_{\mathrm{k}}, J_{k}=\frac{\partial \operatorname{vec}\left(\mathcal{H}_{B T D}\right)}{\partial Z^{T}}$, zero vector and identity matrix have apposite dimensions. Jacobian is rank scarce due to scaling indeterminacy of decomposition. As a result some singular values of jacobian are zero. Due to norm constraint regularization in each step jacobian is singular inLevenbergMarquardt whereas it is necessary to capsize it in an evocative way in Gauss-Newton trust region algorithm. Non-linear least square is considered to be good competitor for alternating least square method [20]. NLS method has been used as optimization method for all the tensor decomposition models in this paper.

\section{RELATED WORK}

The main objective of hyperspectral imaging is identification and segregation of materials based on the unique reflective properties exhibited by them [9].Extracting useful information from a hyperspectral image is a challenging task now days. The basic idea to extract information from the hyperspectral image is to find the relationship between known and unknown elements [3].Different approaches to accomplish the objective include commodity cluster based parallel data analysis strategy which is an unsupervised technique. It integrates spatial and spectral data using multichannel morphological transformations. [9].In [7] a fuzzy segmentation model combined with denoising model was used to detect materials from the image cube. In [6] non-negative factorization based approach was designed to process hyperspectral image which reduces a 3 mode tensor to three non-negative factor matrices. In[16]multi-channel and multi component scheme was proposed which represents the image as tensor and then decomposing it to get the noise free image. Main objective of the scheme was to minimize the mean squared error between original tensor and estimated tensor using filters along each dimension. Tensor CUR decomposition is used to compress hyperspectral image and to reconstruct missing entries using user-product-product preference tensor [11].Additive morphological decomposition was proposed in [17] which define scale space decomposition for multidimensional images without any loss of information. In [18] an anomaly detection method was proposed using the concept of coskewness tensor. It produces a single detection map without any iteration. Processing hyperspectral image using tensor analysis is getting more and more attention these days because using tensor one can integrate spatial as well as spectral domain to extract information of interest from HSI, unlike traditional methods used for its analysis. 


\section{RESULTS}

This section deals with performance evaluation of the tensor decomposition models explained in section 2.Fourtensor decomposition models namely LMLRA, BTD $\left(\mathrm{L}_{\mathrm{r}}, \mathrm{L}_{\mathrm{r}}, 1\right), \mathrm{BTD}(\mathrm{L}, \mathrm{M}, \mathrm{N})$ and $\mathrm{BTD}(\mathrm{L}, \mathrm{M},-)$ were used to reduce the given image cube into low rank image. A hyperspectral image of size $(255,335,33)$ is modeled as a tensor of mode 3.Each models deals with selection of single core tensor or more than one tensor. The size of core tensors was chosen arbitrarily based on the type of decomposition models. The effectiveness of the models is decided on the basis of frobenius norm value and the relative errors generated during iterations. The algorithms used are designed in a way such that best result could be achieved by undergoing iterations until convergence to achieve it. Different levels of noise were added to tensor to check the tolerance of models.

\subsection{LMLRA}

Low multi-linear rank approximation (LMLRA) model decomposes original tensor into core tensor of low rank than original tensor and factor matrices. Algorithm used for computing LMLRA is non-linear least square method. Size of core tensor is $(127,167,16)$ is chosen.Frobenius norm value predicts the dissimilarity between original tensor and generated tensor. The upper bound of this value for four noise levels is same but it is decreasingwith increase in iterations which signify the tolerance level of the model under different noise levels. The frobenius norm value graph for LMLRA is shown in figure1.

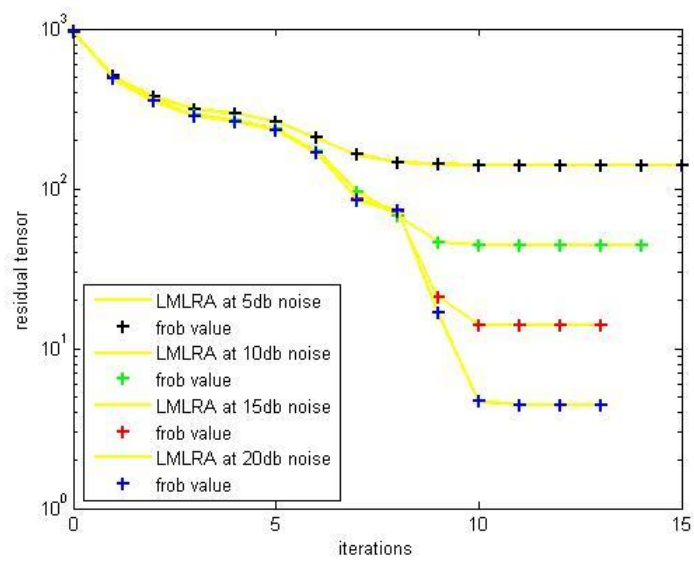

Fig 1: Frobenius norm value graph generated using LMLRA tensor model

\section{2 $\mathrm{BTD}\left(\mathrm{L}_{\mathrm{r}}, \mathrm{L}_{\mathrm{r}}, 1\right)$}

It is a combination of tucker decomposition and canonical polyadic decomposition. Tucker tensor model decomposes a tensor into core tensor and factor matrices and CPD decomposes original tensor into sum of rank-1 tensors.Block Term Decomposition approximates the original tensor into sum of multi-linear rank terms i.e. series of core tensors and factor matrices. We have selected three core tensors of size $([200,200,1],[100,100,1],[50,50,1])$. Non-linear least square algorithm is used to compute block term decomposition. Upperbounds frobenius value are different for all noise levels which makes it fit for highly noised level data and also the values are far less than LMLRA.The decomposition method works well for the image cube having good spatial resolution.Figure 2 shows the corresponding graph for the model under different noise levels.

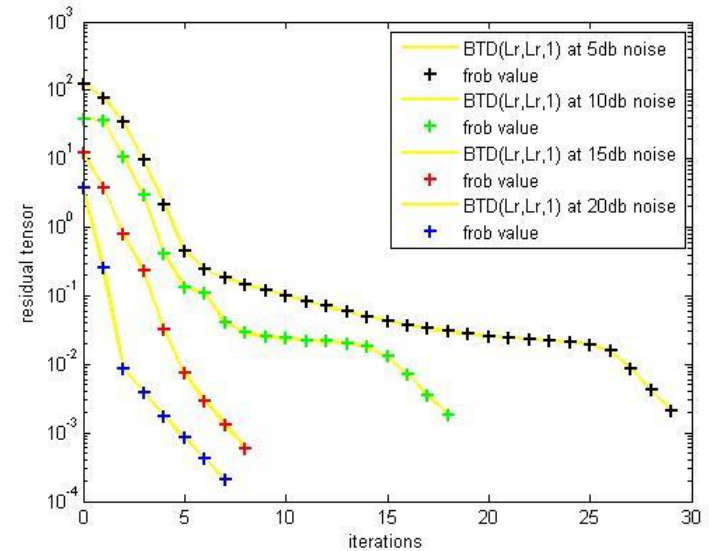

Fig 2:Frobenius norm value graph generated using BTD $\left(\mathrm{L}_{\mathrm{r}}, \mathrm{Lr}, \mathbf{1}\right)$ tensor model

\subsection{BTD (L, M, N)}

For this method also we chose three core tensors of size ([200, $250,25],[100,150,15],[50,100,10])$.The algorithm used to compute BTD is non-linear least square.Although the upper bound frobenius norm value have the same pattern with the BTD $\left(\mathrm{L}_{\mathrm{r}}, \mathrm{L}_{\mathrm{r}}, 1\right)$, the difference lies in the lower values.As inthe above method lower values is having a great difference between tensor mixed with 10 and $15 \mathrm{~dB}$ noise whereas for this methodless fluctuation of values were observed while going down as in the graph.This will work best if the image will have good spectral but poor spatial resolution. Choosing the core tensor is the more critical task for this method. Frobenius value graph for BTD $(\mathrm{L}, \mathrm{M}, \mathrm{N})$ is shown in figure 3.

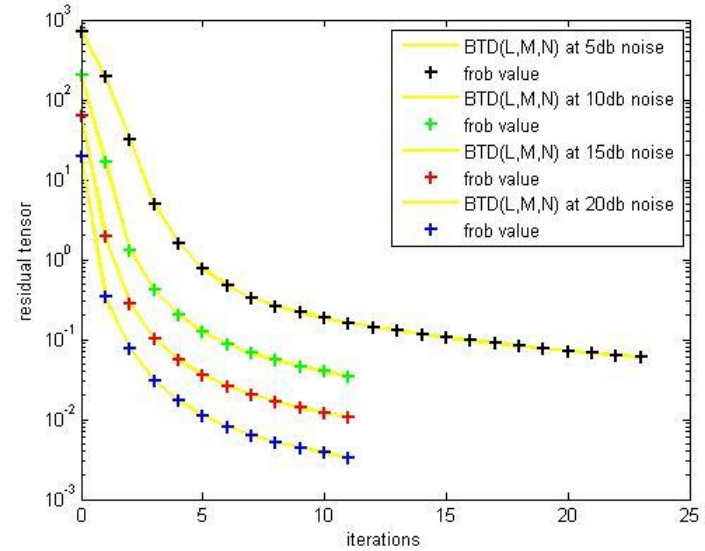

Fig 3:Frobenius norm value graph generated using BTD $(\mathrm{L}, \mathrm{M}, \mathrm{N})$ tensor model

\subsection{BTD (L, M, •)}

This method like all other BTD methods decomposes a tensor in to series of tucker low rank tensors. When all the spectral bands of a hyperspectral image is necessary to extract information of interest from the given cube, this method proves to be the best for this situation. For this method the core tensors of size ([200 250 33], [100 150 33], [ [50 100 33]) has been chosen. Algorithm used is BTD nonlinear least square. This method is the slowest of all for an obvious reason of considering all spectral bands of the image. This methods works good for all levels of noise except for $15 \mathrm{~dB}$ noise but ended with the best value under this noise level. Figure 4 shows the frobenius value graph for $B T D(L, M, \cdot)$. 


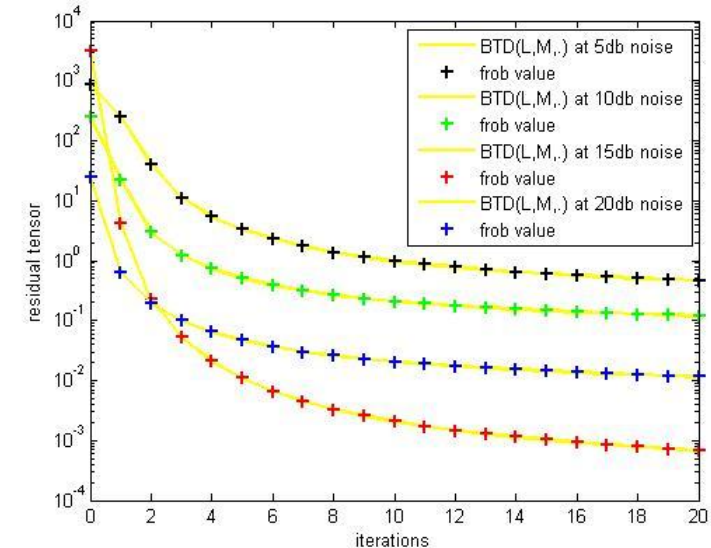

Fig.4.Frobenius norm value graph generated using $B T D$ $(L, M, \cdot)$ tensor model

Table1.Relative Error For Decomposition Models

\begin{tabular}{|c|c|c|c|c|}
\hline $\begin{array}{l}\text { Nois } \\
\text { e } \\
\text { level }\end{array}$ & $\begin{array}{l}\text { LMLR } \\
\text { A }\end{array}$ & $\begin{array}{l}\text { BTD } \\
\left(\mathbf{L}_{\mathbf{r}}, \mathbf{L r}, \mathbf{1}\right)\end{array}$ & $\begin{array}{l}\text { BTD } \\
(\mathbf{L}, \mathbf{M}, \mathbf{N})\end{array}$ & $\begin{array}{l}\text { BTD (L,M, } \\
-)\end{array}$ \\
\hline $5 \mathrm{~dB}$ & 0.0369 & $1.126 \mathrm{e}-05$ & $5.908 \mathrm{e}-05$ & $3.754 \mathrm{e}-04$ \\
\hline $10 \mathrm{~dB}$ & 0.0117 & $9.709 \mathrm{e}-06$ & $3.442 \mathrm{e}-05$ & $9.525 \mathrm{e}-05$ \\
\hline $15 \mathrm{~dB}$ & 0.0037 & $3.190 \mathrm{e}-06$ & $1.055 \mathrm{e}-05$ & $2.964 \mathrm{e}-05$ \\
\hline $20 \mathrm{~dB}$ & 0.1176 & $1.118 \mathrm{e}-06$ & $3.321 \mathrm{e}-06$ & $9.328 \mathrm{e}-06$ \\
\hline
\end{tabular}

\section{CONCLUSION}

Four methods LMLRA,BTD $\left(\mathrm{L}_{\mathrm{r}}, \mathrm{L}_{\mathrm{r}}, 1\right), \mathrm{BTD}(\mathrm{L}, \mathrm{M}, \mathrm{N})$ and $\mathrm{BTD}(\mathrm{L}, \mathrm{M},-)$ was compared using frobenius norm and relative error value. Based on this value graphs generated for the models $\mathrm{BTD}(\mathrm{Lr}, \mathrm{Lr}, 1)$ upper bound value is less among all models for all noise levels but the limitation of this method is it can only be used for the image cube with good spatial region. $\mathrm{BTD}(\mathrm{L}, \mathrm{M},-)$ have shown the worst performance since the upper bound of frobenius value is close to $10^{4}$ which is worst among all. All models except the earlier model upper bound is close to $10^{3}$.As far as the lower bound of this value for all models are concerned except LMLRA all the models have reached the frobenius value to zero at the fourth significant digit after decimal indicating data effective compression for the image cube. Table 1 shows the relative errors generated during decompositions for all noise levels. It can be seen that LMLRA had performed the worst for all noise levels. Least relative error is generated by BTD $\left(\mathrm{L}_{\mathrm{r}}, \mathrm{Lr}\right.$, 1) for $20 \mathrm{~dB}$ noise whereas the highest relative error is generated by LMLRA at the same level.

It is further concluded that BTD $\left(\mathrm{L}_{\mathrm{r}}, \mathrm{L}_{\mathrm{r}}, 1\right)$ has performed the best for our hyperspectral dataset under all noise levels for the reason that the dataset used have good spatial resolution whereas LMLRA performed the worst for the dataset.

\section{REFERENCES}

[1] Cheng-Chen Lin, Yin-TsungHwang,2011,"Lossless Compression of Hyperspectral Images Using Adaptive Prediction and Backward Search Schemes",Journal Of Information Science And Engineering,Vol.27,pp.419435 .

[2] L. Hitchcock." ,1927, The expression of a tensor or a polyadic as a sum of products", Journal of Mathematical Physics, Vol.6, pp.164-189.
[3] Ji Liu, Przemyslaw Musialski, Peter Wonka, and JiepingYe,2012,"Tensor Completion for Estimating Missing Values in Visual Data", Pattern Analysis and Machine Intelligence, IEEE Transactions, Vol.35, pp.s 208-220, January .

[4] L. R. Tucker,1963, Implications of factor analysis of three-way matrices for measurement of change, in Problems in Measuring Change, C. W. Harris, ed., University of Wiscons in Press, pp. 122-137, 1963.

[5] L. R. Tucker, 1966," Some mathematical notes on threemode factor analysis", Psychometrika, Vol.31, pp.279$311,1966$.

[6] Qiang Zhang, Han Wang, Robert J. Plemmons,V. Pau'l Pauca,2008,"Tensor methods for hyperspectral data analysis: a space object material identification study",J. Opt. Soc. Am. A, Vol.25, (December 2008), pp.30013012.

[7] Fang Lia, Michael K. Ngb, Robert Plemmonsc, Sudhakar Prasadd, Qiang Zhang,2010,"Hyperspectral image segmentation, deblurring, and spectral analysis for material identification", in Proc. SPIE, Visual Information Processing XIX, Vol.7701 770103 (April 2010).

[8] Cichocki, D. Mandic, A-H. Phan, C. Caiafa, G. Zhou, Q. Zhao, and L. De Lathauwer,2015"Tensor Decompositions for Signal Processing Applications: From Two-way to Multi-way Component Analysis", Signal Processing Magazine, IEEE,Vol.32( March 2015)pp.145-163,.

[9] Antonio Plaza, David Valencia, Javier Plaza, Pablo Martinez, 2006,"Commodity cluster-based parallel processing of hyperspectralimagery", J. Parallel Distrib. Comput. Vol.66 (March 2006),pp. 345 - 358,

[10] GaryShaw, DimitrisManolakis, 2002,"Signal Processing for Hyperspectral Image Exploitation" ,IEEE signal processing magzine,pp.12-16.

[11] Michael W. Mahoney, Mauro Maggioni, Petros Drineas,2008,"Tensor CUR Decompositions for Tensor Based Data", SIAM Journal on Matrix Analysis and Applications,Vol.30 (September 2008),pp.957-987.

[12] Tamara G. Kolda,Brett W. Bader,2008,"Tensor Decompositions and Applications", SIAM, Vol.51 (June 2008) ,pp. 455-500.

[13] Z. Pan, G. Healey, M. Prasad, B. Tromberg,2003, "Face recognition in hyperspectral images", IEEE Transactions on Pattern Analysis and Machine Intelligence, Vol.25 (December 2003), pp.1552-1560.

[14] Laurent Sorber, Marc Van Barel and Lieven De Lathauwer,2014, Tensorlab v2.0, Available online, (January 2014)

[15] Salah Bourennane, Caroline Fossati and Alexis Cailly,2010,"Improvement Of Target Detection Based On Tensorial Modelling",18th European Signal Processing Conference,pp.304-308.

[16] Santiago Velasco-Forero, Jesus Angulo,2013, "Classification of hyperspectral images by tensor modeling and additive morphological decomposition" Pattern Recognition, Elsevier, Vol.46 ( February 2013), pp.566-577. 
[17] Xiurui Geng, Kang Sun, Luyan Ji ,Yong chao Zhao,2014,"A High-Order Statistical Tensor Based Algorithm for Anomaly Detection in Hyperspectral Imagery",Scientific reports,,Vol.4.

[18] Chein-I-Chang, 2007," Hyperspectral Data Explotation: Theory and Applications ",Wiley.

[19] Laurent Sorber, Marc Van Barel, Lieven De Lathauwer ,2013,"Optimization-Based Algorithms For Tensor Decompositions: Canonical Polyadic Decomposition,in Rank-(Lr, Lr,1) Terms,And A New Generalization",Siam J. Optim.,Vol.23,pp.695-720.
[20] L. Sorber, M. Van Barel, and L. De Lathauwer, 2012," Unconstrained optimization of real functions in complex variables", SIAM J. Optim., Vol.22 , pp. 879-898.

[21] Lieven De Lathauwer,2008,"Decompositions Of A Higher-Order Tensor In BlockTerms-Part Ii: Definitions And Uniqueness",Siam J. Matrix Anal. Appl., Vol.30, pp.1033-1066. 